\title{
Transition State Analysis of Acid-catalyzed dAMP Hydrolysis
}

\author{
Joe A. B. McCann ${ }^{\S}$ and Paul J. Berti*\$\$ \\ Department of Chemistry ${ }^{\ddagger}$, Department of Biochemistry and Biomedical Sciences ${ }^{\S}$, \\ McMaster University, 1280 Main St. W., Hamilton, ON, L8S 4M1, Canada
}

\section{Supporting Information}

Reference 43. Complete citation

Synthesis of $\left[6-{ }^{15} \mathrm{~N}\right]-,\left[7-{ }^{15} \mathrm{~N}\right]-$, and $\left[9-{ }^{15} \mathrm{~N}\right]$ adenine.

Production of PRPPase, APRTase, and RTRase for dATP synthesis

Synthesis of standard, unlabeled $\alpha-$, and $\beta-M e$ dRMP.

Table S1. NMR characterization of $\alpha-M e-d R M P$.

Figure S1. ${ }^{1} \mathrm{H}$ NMR spectrum of $\alpha-M e-d R M P$.

Table S2. NMR characterization of $\beta-M e-d R M P$.

Figure S2. ${ }^{1} \mathrm{H}$ NMR spectrum of $\beta-M e-d R M P$.

Table S3. Kinetic Parameters for dRMP, $\alpha-M e-d R M P$, and $\beta$-Me-dRMP Solvolysis.

Table S4. Calculated ElEs for $\mathrm{N} 1 \mathrm{H}-1 \rightleftharpoons 2+\mathrm{N} 1 \mathrm{H}, \mathrm{N} 7 \mathrm{H}-5$ in a $\mathrm{D}_{\mathrm{N}}{ }^{\star} \mathrm{P}^{\ddagger}+\mathrm{A}_{\mathrm{N}}$ mechanism

Table S5. Calculated EIEs for $\mathrm{N} 1 \mathrm{H}-3 \rightleftharpoons 4+\mathrm{N} 1 \mathrm{H}, \mathrm{N} 7 \mathrm{H}-5$

Table S6. Calculated KIEs for $\mathrm{N} 1 \mathrm{H}-1 \rightarrow \mathrm{N} 1 \mathrm{H}, \mathrm{N} 7 \mathrm{H}-6^{\ddagger}$ in an $\mathrm{A}_{\mathrm{N}} \mathrm{D}_{\mathrm{N}}$ mechanism.

Table S7. Calculated KIEs for $\mathrm{N} 1 \mathrm{H}-1 \rightarrow \mathrm{N} 1 \mathrm{H}, \mathrm{N} 7 \mathrm{H}-7^{\ddagger}$ in $\mathrm{D}_{\mathrm{N}}{ }^{\ddagger *} \mathrm{~A}_{\mathrm{N}}$ mechanism.

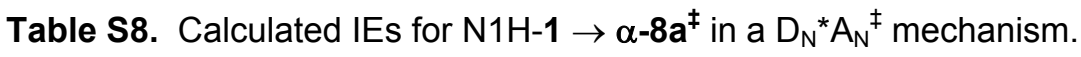

Table S9. Calculated IEs for $\alpha-8 b^{\ddagger}$ in a $D_{N}{ }^{*} A_{N}{ }^{\ddagger}$ mechanism.

Table S10. Calculated IEs for $\beta-8^{\ddagger}$ in a $D_{N}{ }^{*} A_{N}{ }^{\ddagger}$ mechanism.

Table S11. Calculated $11^{1}-{ }^{14} \mathrm{C}$ and ${ }^{15} \mathrm{~N}$ KIEs for $\left(\mathrm{N} 1 \mathrm{H}-\mathbf{1} \rightarrow \mathrm{N} 1 \mathrm{H}, \mathrm{N} 7 \mathrm{H}-\mathbf{7}^{\ddagger}\right)$ and $\left(\mathbf{1} \rightarrow \mathrm{N} 7 \mathrm{H}-\mathbf{7}^{\ddagger}\right)$ for $\mathrm{D}_{\mathrm{N}}{ }^{\ddagger \star} \mathrm{A}_{\mathrm{N}}$ mechanisms.

Figure S3. Effects of $\mathrm{N} 3$ protonation in a $\mathrm{D}_{\mathrm{N}}{ }^{\star} \mathrm{A}_{\mathrm{N}}{ }^{\ddagger}$ mechanism. 
McCann \& Berti

page $\mathrm{S} 2$

TableS12. Summary of the sugar ring geometries.

Table S13. Coordinates for optimized structures.

References.

\section{Reference 43:}

(43) Frisch, M. J.; Trucks, G. W.; Schlegel, H. B.; Scuseria, G. E.; Robb, M. A.; Cheeseman, J. R.; Zakrzewski, V. G.; Montgomery, J. A.; Stratmann, R. E.; Burant, J. C.; Dapprich, S.; Millam, J. M.; Daniels, A. D.; Kudin, K. N.; Strain, M. C.; Farkas, O.; Tomasi, J.; Barone, V.; Cossi, M.; Cammi, R.; Mennucci, B.; Pomelli, C.; Adamo, C.; Clifford, S.; Ochterski, J.; Petersson, G. A.; Ayala, P. Y.; Cui, Q.; Morokuma, K.; Malick, D. K.; Rabuck, A. D.; Raghavachari, K.; Foresman, J. B.; Cioslowski, J.; Ortiz, J. V.; Stefanov, B. B.; Liu, G.; Liashenko, A.; Piskorz, P.; Komaromi, I.; Gomperts, R.; Martin, R. L.; Fox, D. J.; Keith, T.; AlLaham, M. A.; Peng, C. Y.; Nanayakkara, A.; Gonzalez, C.; Challacombe, M.; Gill, P. M. W.; Johnson, B. G.; Chen, W.; Wong, M. W.; Andres, J. L.; Head-Gordon, M.; Replogle, E. S.; Pople, J. A. Gaussian 98), Gaussian, Inc.: Pittsburgh, PA, 1998.

\section{Synthesis of $\left[6-{ }^{15} \mathrm{~N}\right]-,\left[7-{ }^{15} \mathrm{~N}\right]-$, and $\left[9-{ }^{15} \mathrm{~N}\right]$ adenine.}

$\left[6^{15}{ }^{15} \mathrm{~N}\right.$ Adenine. $\left[6{ }^{15} \mathrm{~N}\right]$ adenine was prepared by combining 6-chloropurine, $(0.66 \mathrm{mmol})$, ${ }^{15} \mathrm{NH}_{4} \mathrm{Cl}(1.38 \mathrm{mmol})$, and $\mathrm{KHCO}_{3}(2 \mathrm{mmol})$ in $1 \mathrm{~mL}$ DMSO at $80{ }^{\circ} \mathrm{C}$ for $48 \mathrm{~h} .{ }^{1}$ The cooled suspension was filtered through a sintered glass filter, and DMSO was removed under vacuum at $40{ }^{\circ} \mathrm{C}$. The solid was dissolved in $50 \mathrm{~mL}$ of $80{ }^{\circ} \mathrm{C}$ water, cooled, and extracted overnight with $500 \mathrm{~mL}$ diethyl ether. The aqueous phase was filtered and lyophilized, redissolved in water and purified by C18 reverse-phase HPLC (Waters Delta Pak C18, $7.8 \times 300 \mathrm{~mm}, 15 \mu \mathrm{m}$ particles) in 95:5 water:methanol $(\mathrm{MeOH})$ at $5 \mathrm{~mL} / \mathrm{min}$ to give $\left[6-{ }^{15} \mathrm{~N}\right]$ adenine $(0.14 \mathrm{mmol}) .{ }^{1} \mathrm{H}$ NMR $(500$ MHz, $d_{6}$-DMSO) $\delta 8.106(\mathrm{~s}, \mathrm{H} 2), 8.080(\mathrm{~s}, \mathrm{H} 8)$, and $7.032\left(\mathrm{~d}, \mathrm{~J}=89.6 \mathrm{~Hz}, \mathrm{NH}_{2}\right) .{ }^{1} \mathrm{H}-{ }^{15} \mathrm{~N}-\mathrm{HMBC}$ cross-peaks (500 MHz, $d_{6}$-DMSO) $\delta$ 7.035-78.5. MS (ESI+) $m / z$ (intensity): $m / z_{\text {calc }}=137.1$; $m / z_{\text {obs }}=136.0(2 \%)$ and $137.0(98 \%)$. 
$\left[7-{ }^{15} \mathrm{~N}\right]$ Adenine. $\left[{ }^{7-}{ }^{15} \mathrm{~N}\right]$ adenine was prepared in $0.3 \%$ yield from 6 -amino-2-thioxo-1,2dihydro-4(3H)-pyrimidinone and $\mathrm{Na}^{15} \mathrm{NO}_{3}$ following the procedure of Pagano et al., ${ }^{1}$ except $\left[7-{ }^{15} \mathrm{~N}\right]$ adenine was purified by HPLC as described above. ${ }^{1} \mathrm{H}-{ }^{15} \mathrm{~N}-\mathrm{HMBC}$ cross-peaks $\delta 8.054-$ 228.0. $\mathrm{MS}(\mathrm{ESI}+) \mathrm{m} / \mathrm{z}$ (intensity): $\mathrm{m} / \mathrm{z}_{\text {calc }}=137.1 ; \mathrm{m} / \mathrm{z}_{\mathrm{obs}}=137.0(100 \%)$.

$\left[9_{-}{ }^{15} \mathrm{~N}\right]$ Adenine. $\left[9-{ }^{15} \mathrm{~N}\right]$ Adenine synthesis was based on previous methods. ${ }^{1,2}$ $\left[6{ }^{15} \mathrm{~N}\right]-4$-chloro-5,6-diaminopyrimidine was prepared from 5-amino-4,6-dichloro-pyrimidine (5.6 mmol), ${ }^{15} \mathrm{NH}_{4} \mathrm{Cl}(11 \mathrm{mmol})$, and $\mathrm{KHCO}_{3}(17 \mathrm{mmol})$ dissolved in DMSO $(8.6 \mathrm{~mL})$ with heating at $80{ }^{\circ} \mathrm{C}$ for $50 \mathrm{~h}$. The brown/orange precipitate was suspended in a small volume of absolute ethanol $\left(80^{\circ} \mathrm{C}\right)$, cooled to $65^{\circ} \mathrm{C}$ and passed through a medium sintered glass filter. The solid was dissolved from the filter with DMSO, which was removed under vacuum at $40{ }^{\circ} \mathrm{C}$ to give $\left[6-{ }^{15} \mathrm{~N}\right]$-4-chloro-5,6-diaminopyrimidine $(4.2 \mathrm{mmol})$. This was used to synthesize $\left[9-{ }^{15} \mathrm{~N}\right] 6$-chloropurine, ${ }^{2}$ then $\left[9-{ }^{15} \mathrm{~N}\right]$ adenine following the procedure above. $\left[9-{ }^{15} \mathrm{~N}\right]$ Adenine (0.48 mmol) was HPLC purified as described above. ${ }^{15} \mathrm{~N}$ NMR (600 MHz, $d_{6}$-DMSO) $\delta 156$ (s, N9). $\mathrm{MS}(\mathrm{ESI}+) \mathrm{m} / z$ (intensity): $\mathrm{m} / \mathrm{z}_{\mathrm{calc}}=137.1 ; \mathrm{m} / \mathrm{z}_{\mathrm{obs}}=136.0(3 \%)$ and $137.0(97 \%)$.

\section{Production of PRPPase, APRTase, and RTRase for dATP synthesis}

Escherichia coli phosphoribosyl pyrophosphate synthetase (PRPPase) was purified from an overexpressing strain generously provided by Bjarne Hove-Jensen (University of Copenhagen). ${ }^{3}$ It was expressed as described previously, ${ }^{4}$ and purified by $20 \%$ ammonium sulfate precipitation from the cell lysate, centrifugation at $9,000 \times \mathrm{g}$, redissolution in $50 \mathrm{mM}$ potassium phosphate, $\mathrm{pH}$ 7.5, $2 \mathrm{mM}$ 2-mercaptoethanol, and $1 \mu \mathrm{g} / \mathrm{mL}$ PMSF, and concentrated to $1.1 \mathrm{mg} / \mathrm{mL}$ by ultrafiltration (Microcon YM10, Millipore). The activity assay was based on the method of Gross et al. ${ }^{5}$ His $_{6}$-tagged Saccharomyces cerevisiae adenine phosphoribosyl transferase (APRTase) was purified from an overexpressing strain of $E$. coli generously provided by Vern Schramm (Albert Einstein College of Medicine). ${ }^{6}$ Purification was modified to use only a $\mathrm{Ni}^{2+}$ affinity chromatography, then exchange into $50 \mathrm{mM}$ Tris- $\mathrm{HCl}, \mathrm{pH} 7.4,5 \mathrm{mM} \mathrm{MgCl} 2,20 \mathrm{mM}$ $\mathrm{KCl}$, and $2 \mu \mathrm{g} / \mathrm{mL}$ PMSF and concentration to $1.1 \mathrm{mg} / \mathrm{mL}$. Activity was determined as for PRPPase, but with $3.2 \mathrm{mM}$ adenine and $1 \mathrm{mM}$ phosphoribosyl pyrophosphate in place of ribose-5-phosphate and ATP. Lactobacillus leichmannii ribonucleoside triphosphate reductase 
(RTRase) was purified from an overproducing strain of $E$. coli generously provided by JoAnne Stubbe (Massachusetts Institute of Technology). The protein was purified as described previously, ${ }^{7}$ except the only chromatographic step was cation exchange using SP-Sepharose (GE Healthcare) using a 0 to $1 \mathrm{M} \mathrm{NaCl}$ gradient in $100 \mathrm{mM}$ Tris- $\mathrm{HCl}, \mathrm{pH} 7.5$, at $15 \mathrm{~mL} / \mathrm{min}$. Fractions containing activity were pooled and exchanged into $50 \mathrm{mM}$ Na-HEPES, pH 7.5, $1 \mathrm{M}$ sodium acetate, $1 \mathrm{mM}$ EDTA, $30 \mathrm{mM}$ dithiothreitol (DTT), $1 \mathrm{mM} \mathrm{ATP}$, and $8 \mu \mathrm{M}$ coenzyme B12, and concentrated to $30 \mathrm{mg} / \mathrm{mL}$. All purified proteins were stored at $-20^{\circ} \mathrm{C}$.

\section{Synthesis of standard, unlabeled $\alpha-$, and $\beta$-methyl dRMP}

$\alpha$-, and $\beta$-Me dRMP's ( $\alpha$-, and $\beta$-methyl 2'-deoxy-D-ribose-5'-monophosphate) were synthesized from $10 \mathrm{mg}$ dAMP in $24 \mathrm{~mL} \mathrm{MeOH} / 0.1 \mathrm{~N} \mathrm{HCl}$ for $48 \mathrm{~h}$ at $30^{\circ} \mathrm{C}$. The solution was neutralized with $\mathrm{NaOH}$. To this was added $37 \mathrm{mM}$ potassium phosphate, and $37 \mathrm{mM}$ TEAA, $\mathrm{pH}$ 6.0. The samples were lyophilized, redissolved in water, and purified as for the ${ }^{14} \mathrm{C}$-labeled compounds. The purified compounds were lyophilized, redissolved in $\mathrm{D}_{2} \mathrm{O}$, and analyzed by ${ }^{1} \mathrm{H}$ NMR. $\alpha$-Me-dRMP eluted at $6.7 \mathrm{~min}$ (Table S1), while $\beta$-Me-dRMP eluted at $8.8 \mathrm{~min}$ (Table S2).

Table S1. NMR characterization of a-Me-dRMP.

\begin{tabular}{|c|c|c|c|c|c|}
\hline \multirow[b]{2}{*}{ atom } & \multicolumn{2}{|c|}{$\delta \mathrm{H}(\mathrm{ppm})$} & \multirow[b]{2}{*}{$\mathrm{J}(\mathrm{H}, \mathrm{H})$} & \multicolumn{2}{|c|}{$\mathrm{J}(\mathrm{Hz})$} \\
\hline & as isolated ${ }^{a}$ & literature $^{b}$ & & as isolated & literature $^{b}$ \\
\hline \multirow[t]{2}{*}{$\mathrm{H}^{\prime}{ }^{\prime}$} & 5.109 & 5.161 & $\left(H 1^{\prime}, \mathrm{H} 2^{\prime} \mathrm{S}\right)$ & 5.5 & 5.4 \\
\hline & & & $\left(\mathrm{H}^{\prime}{ }^{\prime}, \mathrm{H} 2^{\prime} R\right)$ & 1.1 & 1.3 \\
\hline \multirow[t]{3}{*}{ H3' } & 4.268 & 4.245 & $\left(\mathrm{H}^{\prime}, \mathrm{H} 2^{\prime} \mathrm{S}\right)$ & 7.2 & 7.5 \\
\hline & & & $\left(\mathrm{H}^{\prime}, \mathrm{H} 2^{\prime} \mathrm{R}\right)$ & 2.4 & 2.5 \\
\hline & & & $\left(\mathrm{H} 3^{\prime}, \mathrm{H} 4^{\prime}\right)$ & 3.0 & 3.6 \\
\hline $\mathrm{H} 4^{\prime}$ & 4.115 & 4.066 & $\left(\mathrm{H} 4^{\prime}, \mathrm{H} 3^{\prime}\right)$ & 3.5 & 3.6 \\
\hline $\mathrm{H} 5$ ' $R$ and $\mathrm{H} 5$ 'S & $3.899^{c}$ & $\begin{array}{l}3.704 \\
3.620^{d}\end{array}$ & - & - & - \\
\hline $\mathrm{OCH}_{3}$ & 3.304 & 3.371 & - & - & - \\
\hline \multirow[t]{2}{*}{$\mathrm{H} 2$ 'S } & 2.313 & 2.327 & $\left(\mathrm{H} 2^{\prime} \mathrm{S}, \mathrm{H} 2^{\prime} R\right)$ & 14.4 & - \\
\hline & & & (H2'S, H3') & 7.2 & 7.5 \\
\hline
\end{tabular}


McCann \& Berti

page S5

\begin{tabular}{ccccc} 
& & $\left(\mathrm{H} 2^{\prime} \mathrm{S}, \mathrm{H} 1^{\prime}\right)$ & 5.4 & 5.4 \\
$\mathrm{H} 2^{\prime} R$ & \multirow{3}{*}{1.827} & $\left(\mathrm{H} 2^{\prime} \mathrm{R}, \mathrm{H} 2^{\prime} \mathrm{S}\right)$ & 14.3 & - \\
& & $\left(\mathrm{H} 2^{\prime} \mathrm{R}, \mathrm{H} 3^{\prime}\right)$ & 2.2 & 2.5 \\
& & $\left(\mathrm{H} 2^{\prime} \mathrm{R}, \mathrm{H} 1^{\prime}\right)$ & 1.2 & 1.3 \\
\hline
\end{tabular}

a Chemical shifts were internally referenced to the HOD solvent signal. Measured at $300 \mathrm{~K}$.

$b \quad$ Literature values from reference ${ }^{8}$. Measured at $292 \mathrm{~K}$ in $\mathrm{D}_{2} \mathrm{O}$.

c Multiplets corresponding to H5'S and H5'R were not resolved. The chemical shift reported was for the center of the multiplet.

${ }^{d}$ Chemical shifts for H5'S and H5'R of $\alpha$-methyl 2'-deoxy-D-ribose.

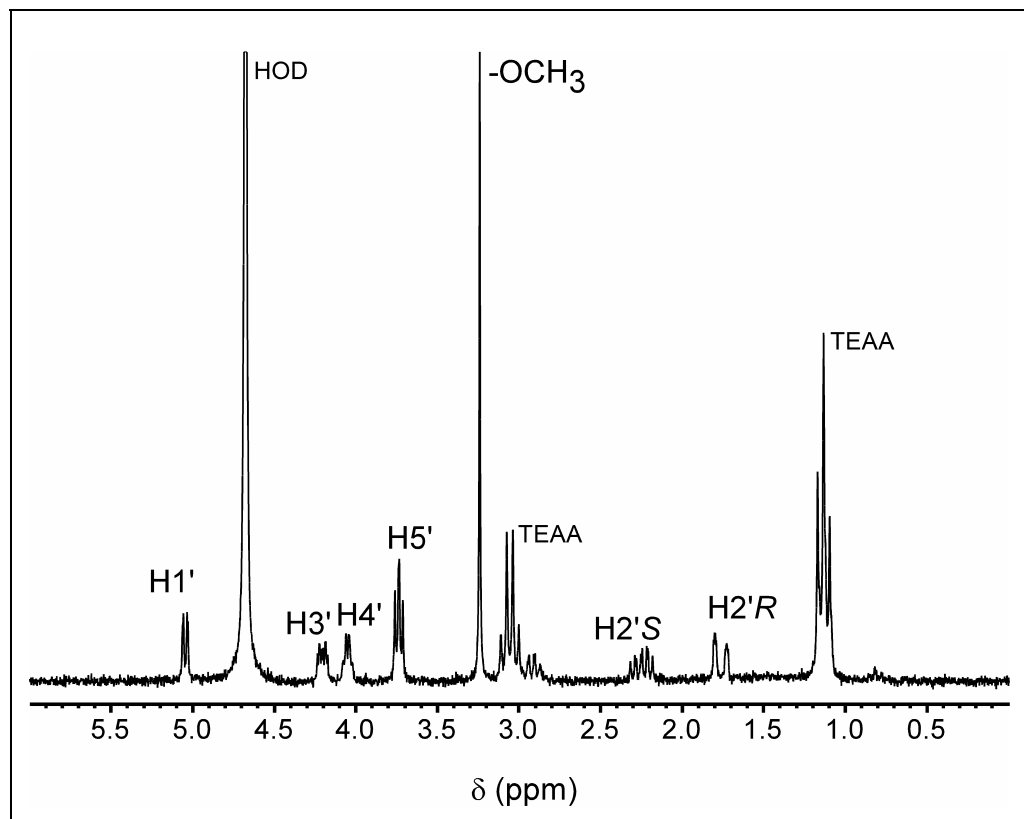

Figure S1. ${ }^{1} \mathrm{H}$ NMR spectrum of $\alpha-M e-d R M P$.

Table S2. NMR characterization of $\beta-M e-d R M P$.

\begin{tabular}{|c|c|c|c|c|c|}
\hline \multirow[b]{2}{*}{ Atom } & \multicolumn{2}{|c|}{$\delta \mathrm{H}(\mathrm{ppm})$} & \multirow[b]{2}{*}{$\mathrm{J}(\mathrm{H}, \mathrm{H})$} & \multicolumn{2}{|c|}{$\mathrm{J}(\mathrm{Hz})$} \\
\hline & as isolated ${ }^{a}$ & literature $^{b}$ & & as isolated & literature \\
\hline \multirow[t]{2}{*}{$\mathrm{H} 1^{\prime}$} & 5.146 & 5.20 & $\left(\mathrm{H} 1^{\prime}, \mathrm{H}^{\prime} \mathrm{R}\right)$ & 5.1 & 5.1 \\
\hline & & & $\left(\mathrm{H} 1^{\prime}, \mathrm{H} 2^{\prime} \mathrm{S}\right)$ & 2.9 & 2.9 \\
\hline \multirow[t]{3}{*}{ H3' } & 4.361 & 4.44 & $\left(\mathrm{H}^{\prime}, \mathrm{H} 2^{\prime} \mathrm{S}\right)$ & 6.3 & 6.6 \\
\hline & & & $\left(\mathrm{H} 3^{\prime}, \mathrm{H}^{\prime} \mathrm{R}\right)$ & 6.3 & 5.7 \\
\hline & & & $\left(\mathrm{H}^{\prime}, \mathrm{H}^{\prime}\right)$ & 3.9 & 4.0 \\
\hline $\mathrm{H} 4{ }^{\prime}$ & 4.008 & 4.05 & - & - & - \\
\hline
\end{tabular}


McCann \& Berti

page S6

\begin{tabular}{ccc}
$\mathrm{H} 5{ }^{\prime} R, \mathrm{H} 5{ }^{\prime} \mathrm{S}$ & 3.783 & 3.78 \\
$\mathrm{OCH}_{3}$ & 3.304 & 3.38 \\
$\mathrm{H} 2{ }^{\prime} \mathrm{S}$ & & 2.24 \\
$\mathrm{H} 2^{\prime} \mathrm{R}$ & $2.100^{\circ}$ & 2.15 \\
\hline
\end{tabular}

a Chemical shifts were internally referenced to the HOD solvent signal. Measured at $300 \mathrm{~K}$.

$b$ Literature values from reference ${ }^{9}$. Measured at $303 \mathrm{~K}$ in $\mathrm{D}_{2} \mathrm{O}$.

c Multiplets corresponding to $\mathrm{H} 2$ ' $S$ and $\mathrm{H} 2$ ' $R$ were not resolved.

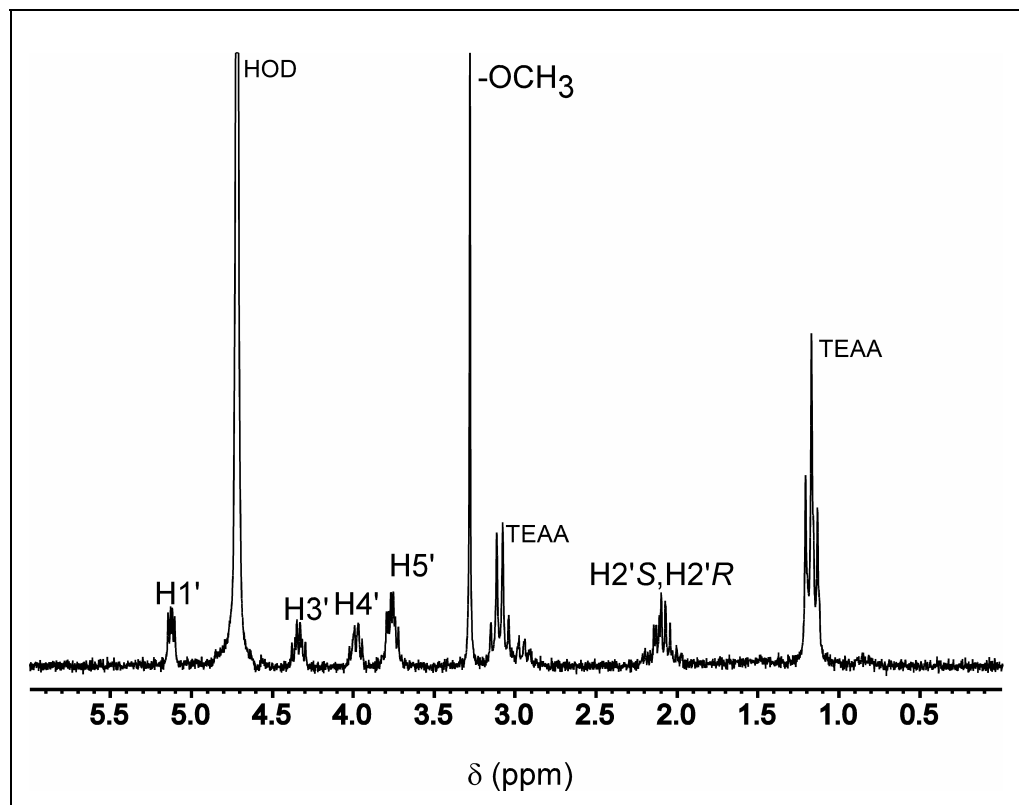

Figure S2. ${ }^{1} \mathrm{H}$ NMR spectrum of $\beta-M e-d R M P$. 
Table S3. Kinetic Parameters for dRMP, $\alpha-M e-d R M P$, and $\beta-M e-d R M P$ Solvolysis. Solvolysis reactions were in $0.1 \mathrm{M} \mathrm{HCl} / 50 \% \mathrm{MeOH}$ at $30{ }^{\circ} \mathrm{C}$. $^{a, b}$

$$
\text { dRMP }
$$

$\alpha-M e-d R M P$

$\beta-M e-d R M P$

$\underline{\mathrm{dRMP}}$ as starting material

\begin{tabular}{|c|c|c|c|}
\hline$k_{6,+n}\left(s^{-1}\right)$ & $2.6 \times 10^{-3}$ & $2.20 \times 10^{-4}$ & $3.1 \times 10^{-4}$ \\
\hline$k_{6,-n}\left(M^{-1} s^{-1}\right)^{c}$ & $2.9 \times 10^{5}$ & $7.9 \times 10^{4}$ & $9.6 \times 10^{4}$ \\
\hline$K_{6, n}($ relative $)$ & 3.2 & 1 & 1.2 \\
\hline$\%$ at Equilibrium ${ }^{d}$ & 13 & 48 & 39 \\
\hline
\end{tabular}

$\alpha-$-Me-dRMP as starting material

\begin{tabular}{|c|c|c|c|}
\hline$k_{6,+n}\left(s^{-1}\right)$ & $1.2 \times 10^{-3}$ & $2.24 \times 10^{-4}$ & $2.5 \times 10^{-4}$ \\
\hline$k_{6,-n}\left(M^{-1} s^{-1}\right)$ & $1.6 \times 10^{5}$ & $1.1 \times 10^{5}$ & $9.7 \times 10^{4}$ \\
\hline$K_{6, n}($ relative $)$ & 3.7 & 1 & 1.3 \\
\hline$\%$ at Equilibrium ${ }^{d}$ & 14 & 46 & 40 \\
\hline
\end{tabular}

$\beta$-dRMP as starting material

\begin{tabular}{|c|c|c|c|}
\hline$k_{6,+n}\left(s^{-1}\right)$ & $1.2 \times 10^{-3}$ & $2.24 \times 10^{-4}$ & $2.5 \times 10^{-4}$ \\
\hline$k_{6,-n}\left(M^{-1} s^{-1}\right)$ & $1.6 \times 10^{5}$ & $9.0 \times 10^{4}$ & $9.0 \times 10^{4}$ \\
\hline$K_{6, n}($ relative $)$ & 3.0 & 1 & 1.1 \\
\hline$\%$ at Equilibrium ${ }^{d}$ & 15 & 47 & 39 \\
\hline
\end{tabular}

Average valuese

\begin{tabular}{|c|c|c|c|}
\hline$k_{6,+n}\left(s^{-1}\right)$ & $1.7( \pm 0.8) \times 10^{-3}$ & $2.23( \pm 0.02) \times 10^{-4}$ & $2.7( \pm 0.3) \times 10^{-4}$ \\
\hline$k_{6,-n}\left(M^{-1} s^{-1}\right)$ & $2.0( \pm 0.8) \times 10^{5}$ & $9.3( \pm 2) \times 10^{4}$ & $9.4( \pm 0.4) \times 10^{4}$ \\
\hline$K_{6, n}($ relative $)$ & $3.3 \pm 0.3$ & 1 & $1.2 \pm 0.1$ \\
\hline$\%$ at Equilibrium ${ }^{d}$ & $14 \pm 1$ & $47 \pm 1$ & $39 \pm 1$ \\
\hline
\end{tabular}

a Values determined from simultaneous fit of two independent experiments.

$b$ Rate constants were found by fitting the data in Figure 1 simultaneously to Scheme 2.

c The initial guesses of $k_{6,+n}$ were arbitrarily set to $10^{5} \mathrm{M}^{-1} \mathrm{~s}^{-1}$ (rather than the expected values of $\sim 10^{11} \mathrm{M}^{-1} \mathrm{~s}^{-1}$ ), then fitted to Scheme 2 . The fitting software could not handle a $10^{14}$-fold range between $k_{6,-n}$ and $k_{6,+n}$. The $k_{6,+n}$ values were not well determined under the experimental conditions, so the absolute values have no significance beyond being $>>k_{6,-n}$. Their values relative to each other were well-determined, though, and were used to calculate $K_{6, n}($ relative $) . K_{6, n}($ relative $)=K_{6, n} / K_{6, a}$, and $K_{6, n}=$ $k_{6,+n} / k_{6,-n}$.

d Mean of the final product concentrations after $300 \mathrm{~min}$.

e Error is the standard deviation of the three analyses. 
Table S4. Calculated ElEs for $\mathrm{N} 1 \mathrm{H}-1 \rightleftharpoons 2+\mathrm{N} 1 \mathrm{H}, \mathrm{N} 7 \mathrm{H}-5$ in a $\mathrm{D}_{\mathrm{N}}{ }^{*} \mathrm{P}^{\ddagger}+\mathrm{A}_{\mathrm{N}}$ mechanism

\begin{tabular}{ccccc} 
isotopic label & $Q(\mathrm{~N} 1 \mathrm{H}-1)^{a}$ & $Q(2)^{a}$ & $Q(\mathrm{~N} 1 \mathrm{H}, \mathrm{N} 7 \mathrm{H}-5)^{a}$ & $\mathrm{ElE}^{b}$ \\
\hline $1{ }^{14}{ }^{14} \mathrm{C}$ & 1.316 & 1.326 & & 0.993 \\
$9-{ }^{15} \mathrm{~N}$ & 1.122 & & 1.097 & 1.023 \\
$7-{ }^{15} \mathrm{~N}$ & 1.096 & & 1.111 & 0.987 \\
$1{ }^{3}{ }^{3} \mathrm{H}$ & 37.13 & 27.40 & & 1.364 \\
$2{ }^{\prime}{ }^{2}{ }^{2} \mathrm{H}$ & 11.35 & 9.61 & & 1.183 \\
$2{ }^{\prime} R-{ }^{2} \mathrm{H}$ & 10.97 & 10.23 & & 1.078 \\
$6-{ }^{15} \mathrm{~N}$ & 1.096 & & 1.094 & 1.001 \\
$5{ }^{\prime} S, 5^{\prime} R-{ }^{3} \mathrm{H}_{2}$ & 958.1 & 945.3 & & 1.038 \\
\hline
\end{tabular}

a $Q$, reduced isotopic partition functions were calculated using QUIVER $^{10}$ at $303 \mathrm{~K}$.

b $\mathrm{EIE}(\mathrm{N} 1 \mathrm{H}-1=2+\mathrm{N} 1 \mathrm{H}, \mathrm{N} 7 \mathrm{H}-\mathbf{5})=Q(\mathrm{~N} 1 \mathrm{H}-\mathbf{1}) / Q(\mathbf{x})$, where $\mathbf{x}=\mathbf{2}$ or $\mathrm{N} 1 \mathrm{H}, \mathrm{N} 7 \mathrm{H}-\mathbf{5}$.

Table S5. Calculated EIEs for $\mathrm{N} 1 \mathrm{H}-3=4+\mathrm{N} 1 \mathrm{H}, \mathrm{N} 7 \mathrm{H}-5$

\begin{tabular}{cccc} 
isotopic label & $Q(\mathrm{~N} 1 \mathrm{H}-3)^{a}$ & $Q(4)^{a}$ & $\mathrm{ElE}^{b}$ \\
\hline $1{ }^{1}-{ }^{14} \mathrm{C}$ & 1.317 & 1.326 & 0.993 \\
$9-{ }^{15} \mathrm{~N}$ & 1.122 & & 1.023 \\
$7-{ }^{15} \mathrm{~N}$ & 1.096 & & 0.987 \\
$1{ }^{3} \mathrm{H}$ & 37.19 & 27.27 & 1.364 \\
$2{ }^{\prime} \mathrm{S}-{ }^{2} \mathrm{H}$ & 11.28 & 9.53 & 1.183 \\
$2{ }^{2}{ }^{2} \mathrm{H}$ & 11.00 & 10.20 & 1.078 \\
$6-{ }^{15} \mathrm{~N}$ & 1.096 & & 1.001 \\
$5{ }^{\prime} S, 5^{\prime} R-{ }^{3} \mathrm{H}_{2}$ & 1009 & 972.1 & 1.038 \\
\hline
\end{tabular}

a $Q$, reduced isotopic partition functions were calculated using QUIVER $^{10}$ at $303 \mathrm{~K}$.

b $\mathrm{EIE}(\mathrm{N} 1 \mathrm{H}-3=4+\mathrm{N} 1 \mathrm{H}, \mathrm{N} 7 \mathrm{H}-5)=Q(\mathrm{~N} 1 \mathrm{H}-3) / Q(\mathbf{x})$, where $\mathbf{x}=\mathbf{4}$ or $\mathrm{N} 1 \mathrm{H}, \mathrm{N} 7 \mathrm{H}-5$. Values of $\mathrm{Q}(\mathrm{N} 1 \mathrm{H}, \mathrm{N} 7 \mathrm{H}-5)$ from Table S4. 
Table S6. Calculated KIEs for $\mathrm{N} 1 \mathrm{H}-1 \rightarrow \mathrm{N} 1 \mathrm{H}, \mathrm{N} 7 \mathrm{H}-6^{\ddagger}$ in an $\mathrm{A}_{\mathrm{N}} \mathrm{D}_{\mathrm{N}}$ mechanism.

\begin{tabular}{cccc} 
& \multicolumn{2}{c}{$\mathrm{N} 1 \mathrm{H}, \mathrm{N} 7 \mathrm{H}-6^{\ddagger}$} & \\
\cline { 2 - 3 } isotopic label & $Q^{\ddagger a}$ & light $v^{*} /^{\text {heavy }} v^{*} b$ & $\mathrm{KIE}^{\mathrm{c}}$ \\
\hline $1{ }^{1}{ }^{14} \mathrm{C}$ & 1.277 & 1.028 & 1.059 \\
$9-{ }^{15} \mathrm{~N}$ & 1.105 & 1.007 & 1.024 \\
$7-{ }^{15} \mathrm{~N}$ & 1.114 & 1.007 & 0.991 \\
$1{ }^{3}-{ }^{3} \mathrm{H}$ & 35.87 & 1.012 & 1.047 \\
$2{ }^{\prime}{ }^{2} \mathrm{H}$ & 10.97 & 1.011 & 1.045 \\
$2{ }^{\prime} R-{ }^{2} \mathrm{H}$ & 11.08 & 1.007 & 0.997 \\
$6-{ }^{15} \mathrm{~N}$ & 1.097 & 1.011 & 1.010 \\
$5{ }^{\prime} S, 5^{\prime} R-{ }^{3} \mathrm{H}_{2}$ & 994.6 & 1.038 & 1.000 \\
\hline
\end{tabular}

a $Q$, reduced isotopic partition functions; and $Q^{\ddagger}$, "defective" reduced isotopic partition function; calculated using QUIVER ${ }^{10}$ at $303 \mathrm{~K}$.

$b \quad$ light $V^{*} /^{\text {heavy }} V^{*}=$ the Teller-Redlich product ratio in QUIVER ${ }^{10}$ output, and represents the contribution from reaction coordinate (imaginary frequency).

c $\left.\quad \mathrm{KIE}\left(\mathrm{N} 1 \mathrm{H}-1 \rightarrow \mathrm{N} 1 \mathrm{H}, \mathrm{N} 7 \mathrm{H}-6^{\ddagger}\right)=Q(\mathrm{~N} 1 \mathrm{H}-1) / Q\left(\mathrm{~N} 1 \mathrm{H}, \mathrm{N} 7 \mathrm{H}-6^{\ddagger}\right) \times\left({ }^{\text {light }} v^{*}\right)^{\text {heavy }} v^{*}\right)_{\mathrm{N} 1 \mathrm{H}, \mathrm{N} 7 \mathrm{H}-6 \ddagger}$. Values of $Q(\mathrm{~N} 1 \mathrm{H}-1)$ from Table S4.

Table S7. Calculated KIEs for $\mathrm{N} 1 \mathrm{H}-1 \rightarrow \mathrm{N} 1 \mathrm{H}, \mathrm{N} 7 \mathrm{H}-7^{\ddagger}$ in a $\mathrm{D}_{\mathrm{N}}^{\ddagger *} \mathrm{~A}_{\mathrm{N}}$ mechanism.

\begin{tabular}{|c|c|c|c|}
\hline \multirow[b]{2}{*}{ isotopic label } & \multicolumn{2}{|c|}{$\mathrm{N} 1 \mathrm{H}, \mathrm{N} 7 \mathrm{H}-7^{\ddagger}$} & \multirow[b]{2}{*}{$\mathrm{KIE}^{c}$} \\
\hline & $Q^{\ddagger a}$ & ${ }^{\text {light }} V^{*} I^{\text {heavy }} V^{*}$ b & \\
\hline $1^{1}-{ }^{14} \mathrm{C}$ & 1.281 & 1.024 & 1.052 \\
\hline $9-{ }^{15} \mathrm{~N}$ & 1.104 & 1.007 & 1.024 \\
\hline $7-{ }^{15} \mathrm{~N}$ & 1.114 & 1.007 & 0.991 \\
\hline $1^{\prime}-{ }^{3} \mathrm{H}$ & 35.27 & 1.015 & 1.068 \\
\hline $2 S^{\prime}{ }^{2} \mathrm{H}$ & 10.91 & 1.007 & 1.047 \\
\hline $2^{\prime} R^{-2} \mathrm{H}$ & 10.98 & 1.008 & 1.007 \\
\hline $6-{ }^{15} \mathrm{~N}$ & 1.097 & 1.009 & 1.008 \\
\hline $5^{\prime} S, 5^{\prime} R-{ }^{3} \mathrm{H}_{2}$ & 998.9 & 1.037 & 0.994 \\
\hline
\end{tabular}

a $Q$, reduced isotopic partition functions; and $Q^{\ddagger}$, "defective" reduced isotopic partition function; calculated using QUIVER ${ }^{10}$ at $303 \mathrm{~K}$.

$b \quad$ light $V^{*} /^{\text {heavy }} V^{*}=$ the Teller-Redlich product ratio in QUIVER ${ }^{10}$ output, and represents the contribution from reaction coordinate (imaginary frequency).

c $\quad \mathrm{KIE}\left(\mathrm{N} 1 \mathrm{H}-1 \rightarrow \mathrm{N} 1 \mathrm{H}, \mathrm{N} 7 \mathrm{H}-7^{\ddagger}\right)=Q(\mathrm{~N} 1 \mathrm{H}-1) / Q\left(\mathrm{~N} 1 \mathrm{H}, \mathrm{N} 7 \mathrm{H}-7^{\ddagger}\right) \times\left({ }^{\text {light }} v^{*} /{ }^{\text {heavy }} v^{*}\right)_{\mathrm{N} 1 \mathrm{H}, \mathrm{N} 7 \mathrm{H}-7 \ddagger}$. Values of $Q(\mathrm{~N} 1 \mathrm{H}-1)$ from Table S4. 


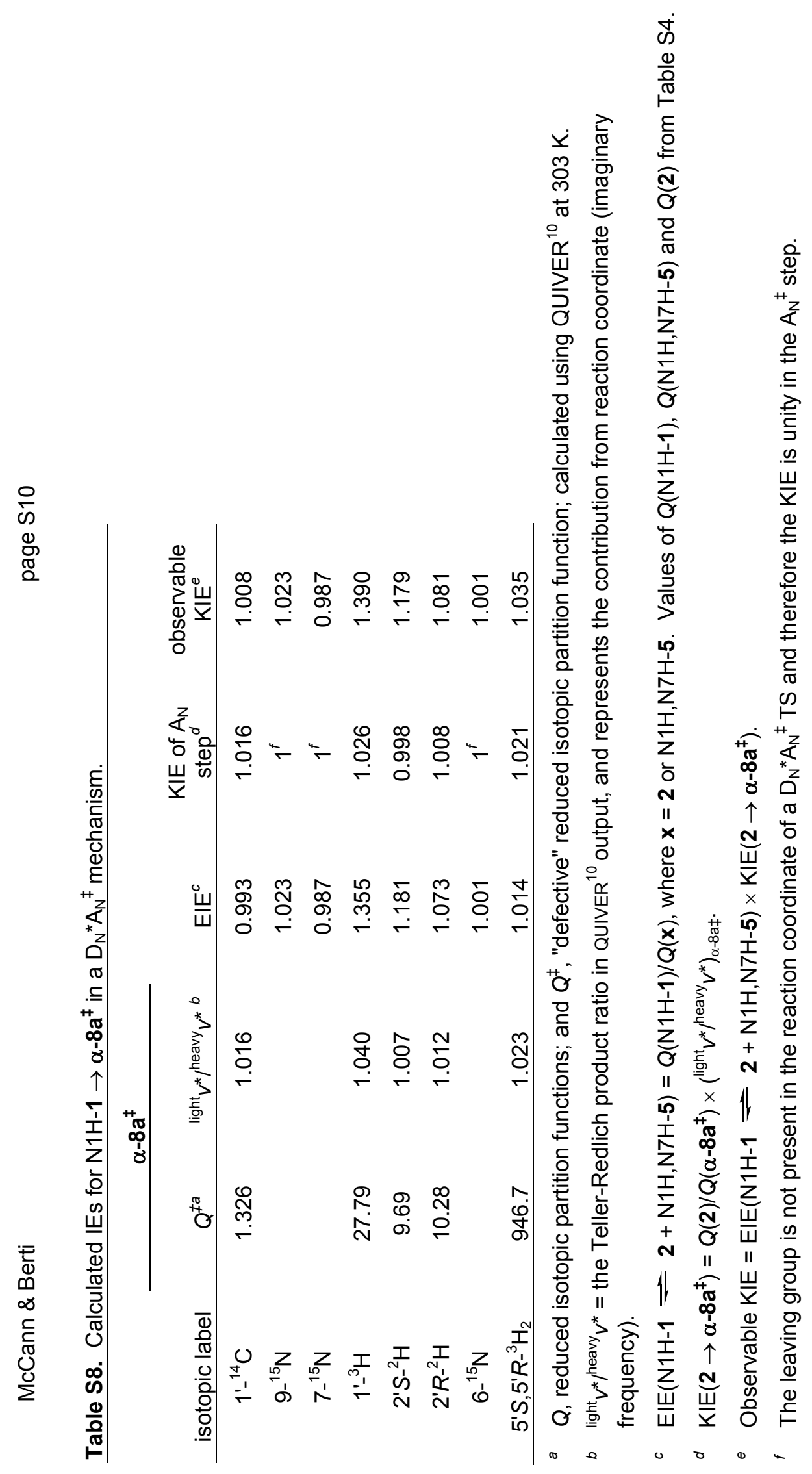




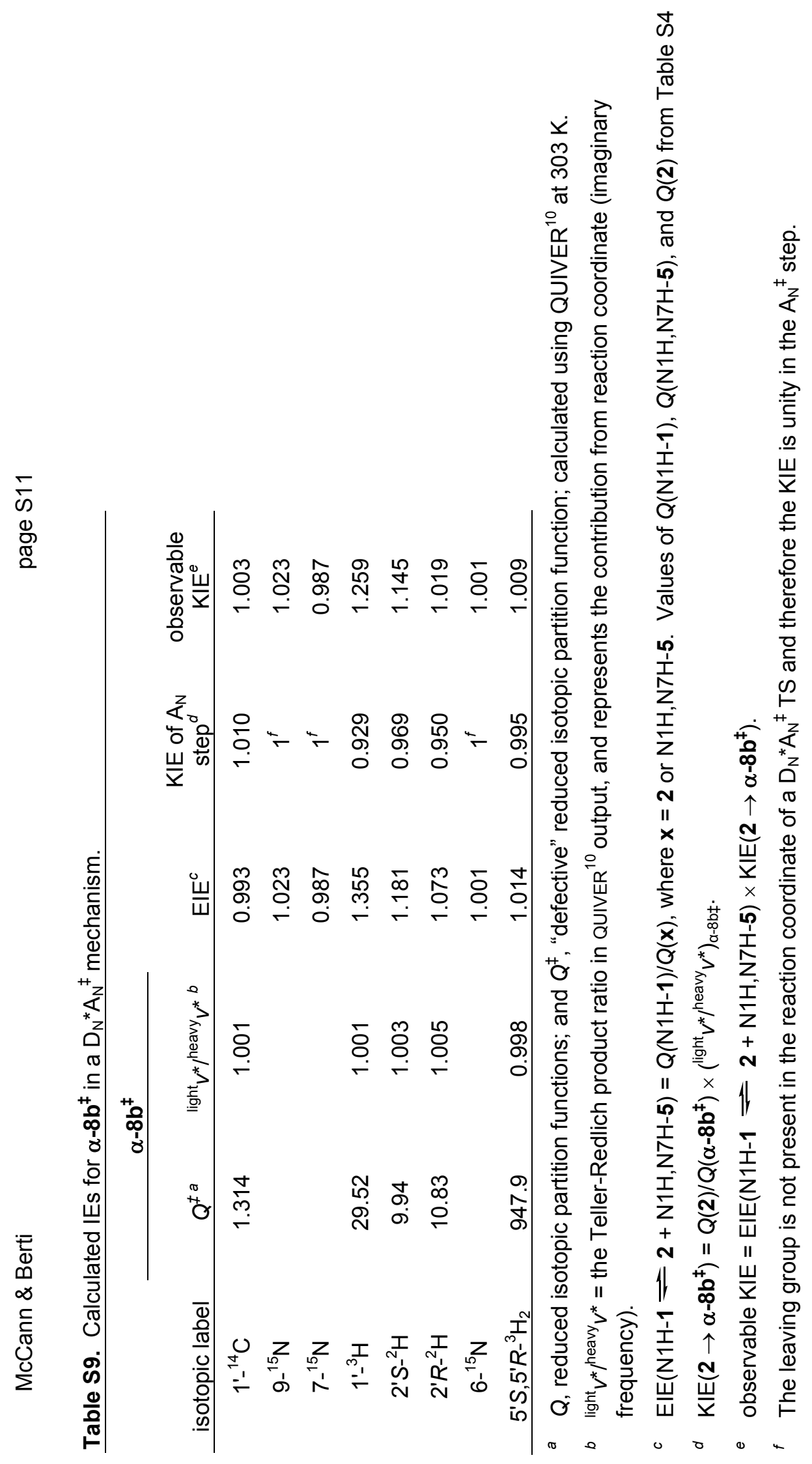




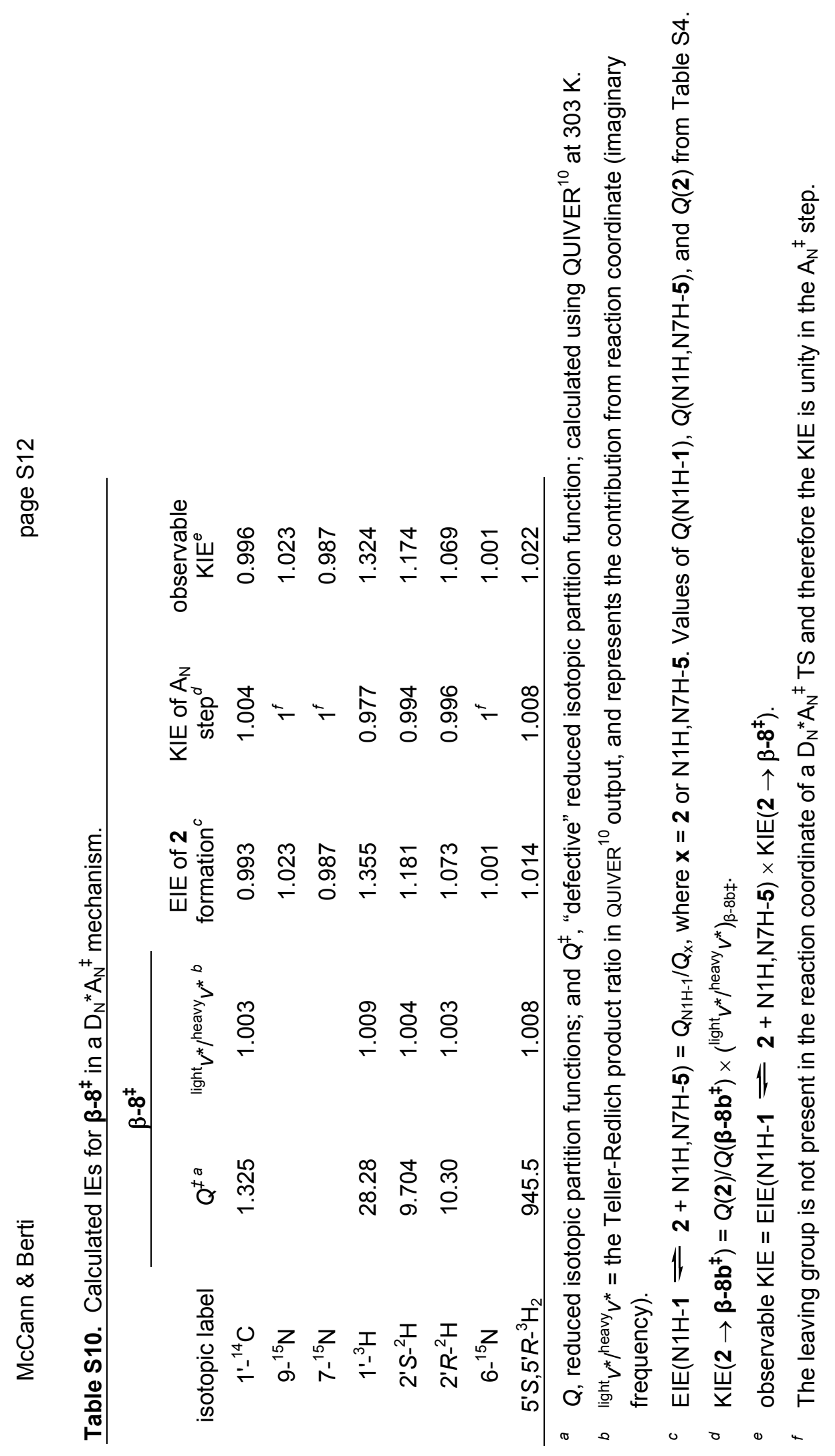


Table S11. Calculated $1{ }^{1-}-{ }^{14} \mathrm{C}$ and ${ }^{15} \mathrm{~N}$ KIEs for $\left(\mathrm{N} 1 \mathrm{H}-\mathbf{1} \rightarrow \mathrm{N} 1 \mathrm{H}, \mathrm{N} 7 \mathrm{H}-\mathbf{7}^{\ddagger}\right)$ and $\left(\mathbf{1} \rightarrow \mathrm{N} 7 \mathrm{H}-7^{\ddagger}\right)$ for $\mathrm{D}_{\mathrm{N}}{ }^{\ddagger \star} A_{N}$ mechanisms.

\begin{tabular}{|c|c|c|c|c|}
\hline \multirow[b]{2}{*}{ isotopic label } & \multirow[b]{2}{*}{$Q(\text { reactant })^{a}$} & \multicolumn{2}{|c|}{ TS } & \multirow[b]{2}{*}{$\mathrm{KIE}^{c}$} \\
\hline & & $Q^{\ddagger a}$ & ${ }^{\text {light }} v^{*} /^{\text {heavy }} v^{*} b$ & \\
\hline \multicolumn{5}{|c|}{$\mathrm{N} 1 \mathrm{H}-1 \rightarrow \mathrm{N} 1 \mathrm{H}, \mathrm{N} 7 \mathrm{H}-7^{\ddagger}$} \\
\hline $1^{\prime}-{ }^{14} \mathrm{C}$ & 1.316 & 1.281 & 1.024 & 1.052 \\
\hline $9-{ }^{15} \mathrm{~N}$ & 1.122 & 1.104 & 1.007 & 1.024 \\
\hline \multicolumn{5}{|l|}{$1 \rightarrow \mathrm{N} 7 \mathrm{H}-7^{\ddagger}$} \\
\hline $1^{\prime}-{ }^{14} \mathrm{C}$ & 1.322 & 1.313 & 1.005 & 1.012 \\
\hline $9-{ }^{15} \mathrm{~N}$ & 1.122 & 1.098 & 1.010 & 1.032 \\
\hline
\end{tabular}

a $Q$, reduced isotopic partition functions; and $Q^{\ddagger}$, "defective" reduced isotopic partition function; calculated using QUIVER ${ }^{10}$ at $303 \mathrm{~K}$.

${ }_{b} \quad$ light $v^{*} /$ heavy $V^{*}=$ the Teller-Redlich product ratio in QUIVER ${ }^{10}$ output, and represents the contribution from reaction coordinate (imaginary frequency).

${ }^{c} \quad \mathrm{KIE}\left(\mathbf{x} \rightarrow \mathbf{y}^{\ddagger}\right)=Q(\mathbf{x}) / Q\left(\mathbf{y}^{\ddagger}\right) \times\left({ }^{\text {light }} v^{*} /{ }^{\text {heavy }} V^{*}\right)_{\mathrm{y}}$, where $\mathbf{x}$ and $\mathbf{y}=\mathrm{N} 1 \mathrm{H}-1$ and $\mathrm{N} 1 \mathrm{H}, \mathrm{N} 7 \mathrm{H}-7^{\ddagger}$ or 1 and $\mathrm{N} 7 \mathrm{H}-7^{\ddagger}$

(a)

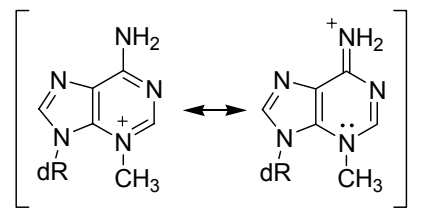

(b)

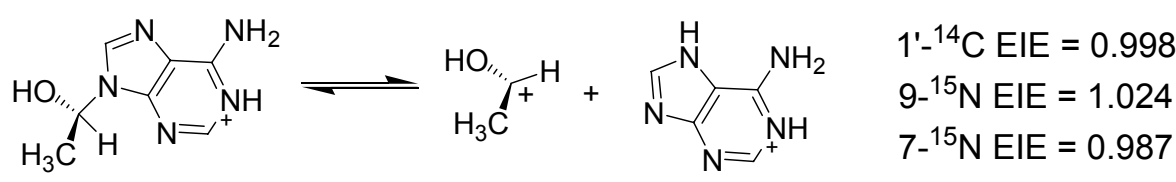

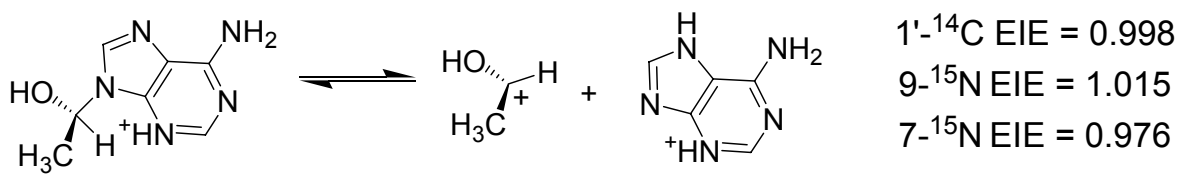

Figure S3. Effects of $\mathrm{N} 3$ protonation in a $\mathrm{D}_{\mathrm{N}}{ }^{*} \mathrm{~A}_{\mathrm{N}}{ }^{\ddagger}$ mechanism. (a) Aromatic and quininoid-like resonance forms of 3-methyl-dAdo. (b) ElEs for the formation of $\mathrm{N} 7 \mathrm{H}, \mathrm{N} 1 \mathrm{H}$-adenine (top) and $\mathrm{N} 7 \mathrm{H}, \mathrm{N} 3 \mathrm{H}$-adenine (bottom). The EIEs on $\mathrm{C}-\mathrm{N}$ bond cleavage equal the observable KIEs for $a \mathrm{D}_{\mathrm{N}}{ }^{*} \mathrm{~A}_{\mathrm{N}}{ }^{\ddagger}$ mechanism. 
McCann \& Berti

page S14

TableS12. Summary of the sugar ring geometries. ${ }^{a}$

\begin{tabular}{|c|c|c|c|c|c|}
\hline Bond & 2 & $\alpha-8 a^{\ddagger}$ & $\beta-8^{\ddagger}$ & $\alpha-8 b^{\ddagger}$ & 9 \\
\hline C1'-O4' & $1.257(1.67)$ & $1.261(1.64)$ & $1.261(1.64)$ & $1.264(1.63)$ & $1.272(1.58)$ \\
\hline C1'-C2' & $1.464(1.23)$ & $1.464(1.23)$ & $1.464(1.23)$ & $1.472(1.20)$ & $1.480(1.17)$ \\
\hline $\mathrm{C} 1^{\prime}-\mathrm{H} 1^{\prime}$ & $1.091(1.00)$ & $1.088(1.01)$ & $1.087(1.01)$ & $1.086(1.01)$ & $1.086(1.01)$ \\
\hline $\mathrm{C} 1{ }^{\prime}-\mathrm{Nu}$ & - & $2.826(0.01)$ & $2.699(0.01)$ & $2.318(0.05)$ & $2.179(0.08)$ \\
\hline$\Sigma \mathrm{n}_{\mathrm{C} 1^{\prime}}$ & (3.89) & (3.89) & (3.90) & (3.89) & $(3.84)$ \\
\hline C2'-H2'S & $1.101(0.97)$ & $1.100(0.97)$ & $1.100(0.97)$ & $1.101(0.97)$ & $1.100(0.97)$ \\
\hline $\mathrm{C} 2^{\prime}-\mathrm{H} 2^{\prime} R$ & $1.097(0.98)$ & $1.096(0.98)$ & $1.095(0.98)$ & $1.091(1.00)$ & $1.090(1.00)$ \\
\hline $\begin{array}{l}\text { Ring Pucker } \\
\qquad\left(\mathrm{P}^{\circ}\right)^{b}\end{array}$ & ${ }_{3} \mathrm{~T}^{4}\left(207^{\circ}\right)$ & ${ }_{3} \mathrm{~T}^{4}\left(208^{\circ}\right)$ & $3^{\prime}-$ exо $\left(200^{\circ}\right)$ & ${ }_{3}^{2} \mathrm{~T} \quad\left(188^{\circ}\right)$ & ${ }_{3}^{2} \mathrm{~T} \quad\left(183^{\circ}\right)$ \\
\hline
\end{tabular}

a Distances in Ångstroms, bond order in parentheses.

$b$ Deoxyribosyl ring puckers are indicated with n-endo and $\mathrm{n}$-exo designations, where atom $\mathrm{n}$ is $\mathrm{C} 1$ ' to $\mathrm{C4}^{\prime}$, or O4', and all ring atoms except $\mathrm{n}$ are in the same plane. In $\mathrm{n}$-endo conformations, $\mathrm{n}$ is displaced toward the 5 '-substituent, while in n-exo conformers it is away from the $5^{\prime}$-substituent. Twist geometries are indicated by the letter $\mathrm{T}$ with superscripted numbers indicating that an atom is above the plane of the ring, or endo, and subscripted numbers indicating that an atom is below the plane of the ring, or exo, e.g., ${ }_{3}^{2} \mathrm{~T}$. Numbers to the left side of $\mathrm{T}$ indicate a major deviation from the plane of the ring; numbers on the right indicate a minor deviation. For symmetric twists the numbers both appear on the left side. For example, ${ }_{3}^{2} \mathrm{~T}$ indicates that $\mathrm{C}^{\prime}$ and $\mathrm{C}^{\prime}$ ' above and below the plane of $\mathrm{C} 1^{\prime}, \mathrm{C}^{\prime}$ ' and $\mathrm{O} 4$ ', and both atoms deviate equally from the plane. The term describing ring orientation is defined by the value calculated for $\mathrm{P}^{\circ}$. $\mathrm{P}^{\circ}$ is calculated as $\tan \mathrm{P}^{\circ}=\frac{\left(\tau_{4}+\tau_{1}\right)-\left(\tau_{3}+\tau_{0}\right)}{2 \tau_{2}(\sin 32+\sin 72)}$, where the torsion angles $(\tau)$ are $\tau_{0}=\mathrm{O}^{\prime}-\mathrm{C} 1^{\prime}, \tau_{1}=\mathrm{C} 1^{\prime}-\mathrm{C} 2^{\prime}, \tau_{2}=\mathrm{C} 2{ }^{\prime}-\mathrm{C} 3^{\prime}, \tau_{3}=\mathrm{C} 3^{\prime}-\mathrm{C} 4^{\prime}$, and $\tau_{4}=\mathrm{C} 4{ }^{\prime}-\mathrm{O} 4^{\prime}$. Joint Commission on Biochemical Nomenclature. Pure Appl. Chem. 1983, 55, 1273-1280.

Table S13. Coordinates for optimized structures. All structures were optimized at the RB3PW91/6-31+G** level.

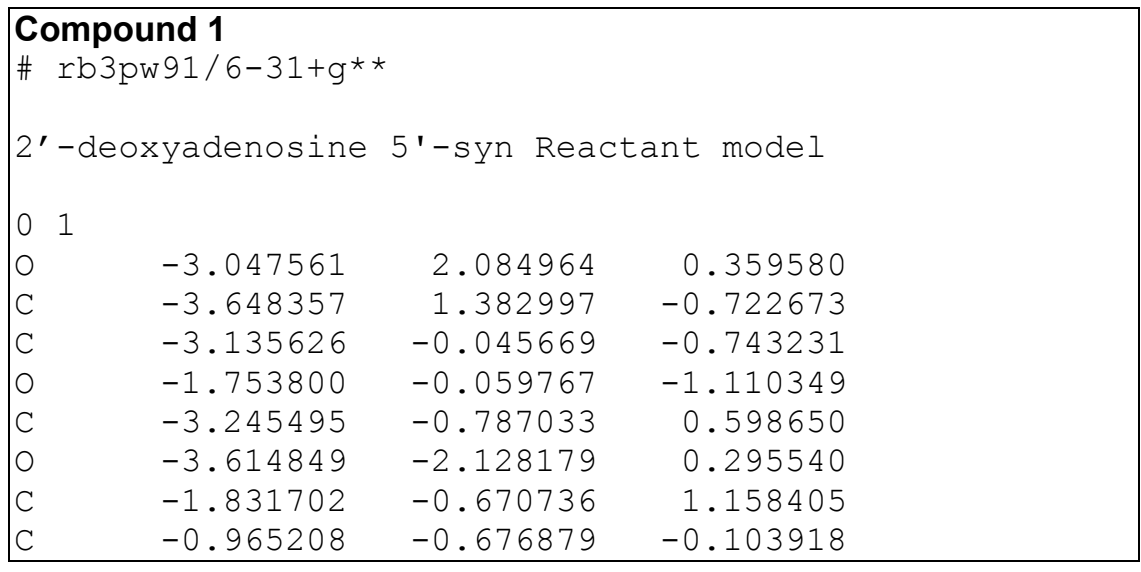


McCann \& Berti

page S15

\begin{tabular}{|c|c|c|c|}
\hline $\mathrm{N}$ & 0.277288 & 0.051900 & 0.024861 \\
\hline C & 0.435868 & 1.407634 & 0.227647 \\
\hline $\mathrm{N}$ & 1.689198 & 1.789774 & 0.275635 \\
\hline C & 2.401260 & 0.621230 & 0.093550 \\
\hline C & 3.779586 & 0.338429 & 0.038792 \\
\hline $\mathrm{N}$ & 4.722928 & 1.294136 & 0.206508 \\
\hline $\mathrm{N}$ & 4.173071 & -0.928913 & -0.168521 \\
\hline C & 3.233780 & -1.875436 & -0.306995 \\
\hline $\mathrm{N}$ & 1.906520 & -1.744820 & -0.269156 \\
\hline C & 1.547578 & -0.472288 & -0.071597 \\
\hline $\mathrm{H}$ & -3.341473 & 3.001733 & 0.341196 \\
\hline $\mathrm{H}$ & -3.398994 & 1.853129 & -1.683370 \\
\hline $\mathrm{H}$ & -4.743743 & 1.360628 & -0.617005 \\
\hline $\mathrm{H}$ & -3.704296 & -0.603513 & -1.498024 \\
\hline $\mathrm{H}$ & -3.989659 & -0.319545 & 1.255900 \\
\hline $\mathrm{H}$ & -3.645278 & -2.636515 & 1.114313 \\
\hline $\mathrm{H}$ & -1.569152 & -1.479313 & 1.846752 \\
\hline $\mathrm{H}$ & -1.722885 & 0.285710 & 1.677642 \\
\hline $\mathrm{H}$ & -0.685049 & -1.692120 & -0.405581 \\
\hline $\mathrm{H}$ & -0.427326 & 2.054707 & 0.312262 \\
\hline $\mathrm{H}$ & 5.685575 & 1.051277 & 0.037583 \\
\hline $\mathrm{H}$ & 4.449399 & 2.262579 & 0.235442 \\
\hline $\mathrm{H}$ & 3.609413 & -2.882919 & -0.472334 \\
\hline \multicolumn{4}{|c|}{$\begin{array}{l}\text { Compound N1H-1 } \\
\# \quad r b 3 p w 91 / 6-31+g(d, p)\end{array}$} \\
\hline \multicolumn{4}{|c|}{ 2'-deoxyadenosine 5'-syn Reactant model } \\
\hline \multicolumn{4}{|c|}{$\begin{array}{ll}1 & 1\end{array}$} \\
\hline O & -2.807334 & 2.124544 & 0.350865 \\
\hline C & -3.512343 & 1.490697 & -0.714904 \\
\hline C & -3.145488 & 0.019996 & -0.757429 \\
\hline 0 & -1.757226 & -0.131264 & -1.114973 \\
\hline C & -3.327655 & -0.743203 & 0.562218 \\
\hline 0 & -3.742443 & -2.055457 & 0.221636 \\
\hline C & -1.921022 & -0.714666 & 1.165278 \\
\hline C & -1.035522 & -0.749790 & -0.082570 \\
\hline $\mathrm{N}$ & 0.228066 & -0.011439 & 0.072311 \\
\hline C & 0.357471 & 1.353399 & 0.241315 \\
\hline $\mathrm{N}$ & 1.606084 & 1.763756 & 0.270411 \\
\hline C & 2.327281 & 0.615386 & 0.104078 \\
\hline C & 3.710324 & 0.413459 & 0.033306 \\
\hline $\mathrm{N}$ & 4.614363 & 1.385609 & 0.137823 \\
\hline $\mathrm{N}$ & 4.074784 & -0.891905 & -0.158620 \\
\hline C & 3.168838 & -1.925947 & -0.275293 \\
\hline N & 1.881122 & -1.781495 & -0.214836 \\
\hline C & 1.490184 & -0.503132 & -0.028638 \\
\hline $\mathrm{H}$ & -3.148955 & 3.018431 & 0.465936 \\
\hline $\mathrm{H}$ & -3.252218 & 1.946853 & -1.678870 \\
\hline $\mathrm{H}$ & -4.598446 & 1.573520 & -0.571761 \\
\hline $\mathrm{H}$ & -3.744255 & -0.466777 & -1.534725 \\
\hline $\mathrm{H}$ & -4.058703 & -0.249383 & 1.213331 \\
\hline $\mathrm{H}$ & -4.024526 & -2.523689 & 1.016115 \\
\hline $\mathrm{H}$ & -1.729160 & -1.554402 & 1.837681 \\
\hline
\end{tabular}


McCann \& Berti

page S16

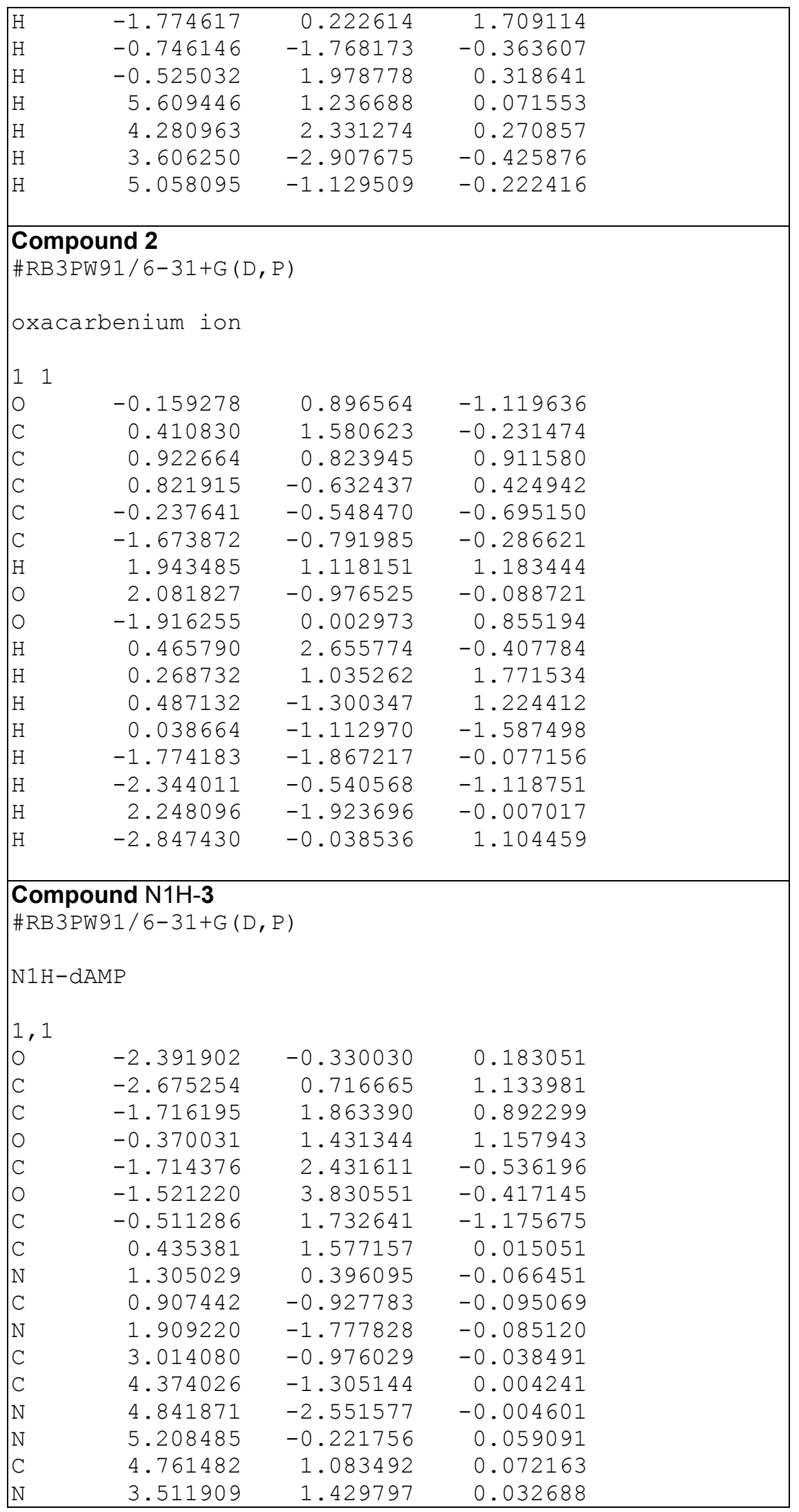


McCann \& Berti

page S17

\begin{tabular}{|c|c|c|c|}
\hline C & 2.663568 & 0.381555 & -0.018740 \\
\hline $\mathrm{H}$ & -2.541846 & 0.318194 & 2.143908 \\
\hline $\mathrm{H}$ & -3.706194 & 1.069230 & 1.013929 \\
\hline $\mathrm{H}$ & -1.961719 & 2.669448 & 1.592975 \\
\hline $\mathrm{H}$ & -2.648467 & 2.201023 & -1.062039 \\
\hline $\mathrm{H}$ & -1.709632 & 4.258300 & -1.260679 \\
\hline $\mathrm{H}$ & -0.069463 & 2.313056 & -1.989063 \\
\hline $\mathrm{H}$ & -0.810327 & 0.752197 & -1.556655 \\
\hline $\mathrm{H}$ & 1.113571 & 2.430862 & 0.121338 \\
\hline $\mathrm{H}$ & -0.141903 & -1.193764 & -0.107864 \\
\hline $\mathrm{H}$ & 5.822155 & -2.783651 & 0.040631 \\
\hline $\mathrm{H}$ & 4.172736 & -3.309553 & -0.037537 \\
\hline $\mathrm{H}$ & 5.540426 & 1.837748 & 0.118451 \\
\hline $\mathrm{H}$ & 6.211109 & -0.367151 & 0.092992 \\
\hline $\mathrm{P}$ & -3.531039 & -1.408989 & -0.163245 \\
\hline 0 & -2.621385 & -2.394871 & -1.048559 \\
\hline $\mathrm{H}$ & -3.107242 & -2.715692 & -1.821102 \\
\hline O & -4.779726 & -0.937820 & -0.797095 \\
\hline 0 & -3.720785 & -2.134469 & 1.259558 \\
\hline $\mathrm{H}$ & -4.626039 & -2.451503 & 1.386741 \\
\hline \multicolumn{4}{|c|}{$\begin{array}{l}\text { Compound 4 } \\
\# \quad r b 3 p w 91 / 6-31+g^{*}\end{array}$} \\
\hline \multicolumn{4}{|c|}{ oxacarbenium ion 5'-phosphate } \\
\hline \multicolumn{4}{|c|}{1,1} \\
\hline 0 & 0.544740 & 0.034793 & 0.018343 \\
\hline C & -0.100889 & -0.721800 & 1.038655 \\
\hline $\mathrm{C}$ & -1.584236 & -0.497362 & 0.873436 \\
\hline 0 & -1.830165 & 0.960879 & 1.172725 \\
\hline C & -2.155076 & -0.666635 & -0.543609 \\
\hline O & -3.466191 & -1.146645 & -0.411059 \\
\hline C & -2.141126 & 0.778339 & -1.100427 \\
\hline C & -2.083824 & 1.591236 & 0.115251 \\
\hline $\mathrm{H}$ & 0.231537 & -0.398408 & 2.031319 \\
\hline $\mathrm{H}$ & 0.101299 & -1.794712 & 0.926093 \\
\hline $\mathrm{H}$ & -2.168790 & -1.038582 & 1.619860 \\
\hline $\mathrm{H}$ & -1.516019 & -1.328217 & -1.136093 \\
\hline $\mathrm{H}$ & -3.717617 & -1.689140 & -1.168637 \\
\hline $\mathrm{H}$ & -3.009876 & 1.028852 & -1.720820 \\
\hline $\mathrm{H}$ & -1.237773 & 1.005756 & -1.688872 \\
\hline $\mathrm{H}$ & -2.235030 & 2.667069 & 0.216777 \\
\hline $\mathrm{P}$ & 2.156625 & -0.115150 & -0.167689 \\
\hline 0 & 2.339031 & 0.968100 & -1.328185 \\
\hline $\mathrm{H}$ & 2.906618 & 0.642430 & -2.041973 \\
\hline O & 2.665403 & -1.473908 & -0.427466 \\
\hline 0 & 2.642845 & 0.585814 & 1.188523 \\
\hline $\mathrm{H}$ & 3.521872 & 0.297268 & 1.474805 \\
\hline \multicolumn{4}{|c|}{$\begin{array}{l}\text { Compound N1H,N7H-5 } \\
\text { \#RB3PW91/6-31+G** }\end{array}$} \\
\hline \multicolumn{4}{|c|}{$\mathrm{N} 1 \mathrm{H}, \mathrm{N} 7 \mathrm{H}$-Adenine } \\
\hline
\end{tabular}


McCann \& Berti

page $\mathrm{S} 18$

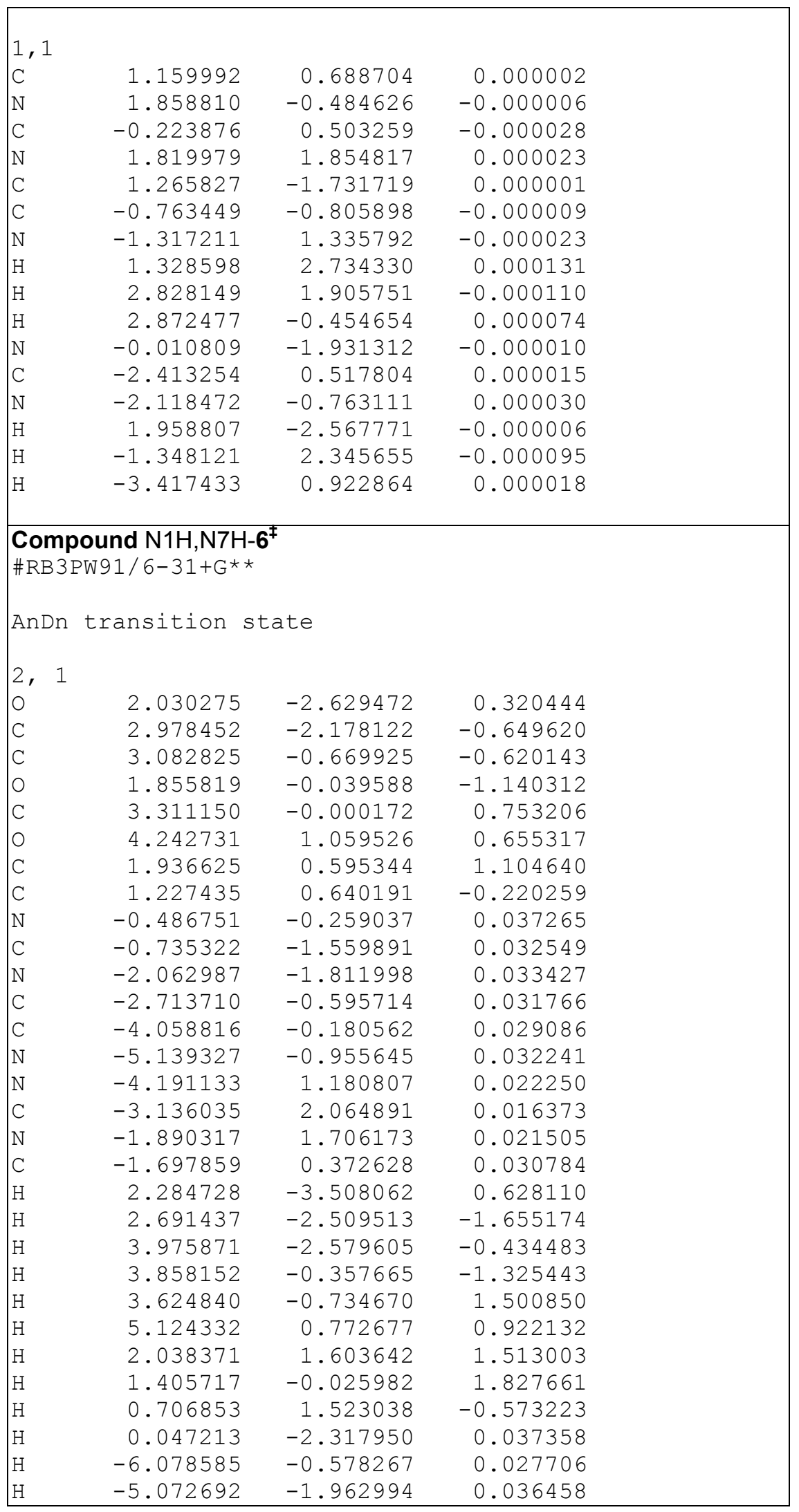


McCann \& Berti

page S19

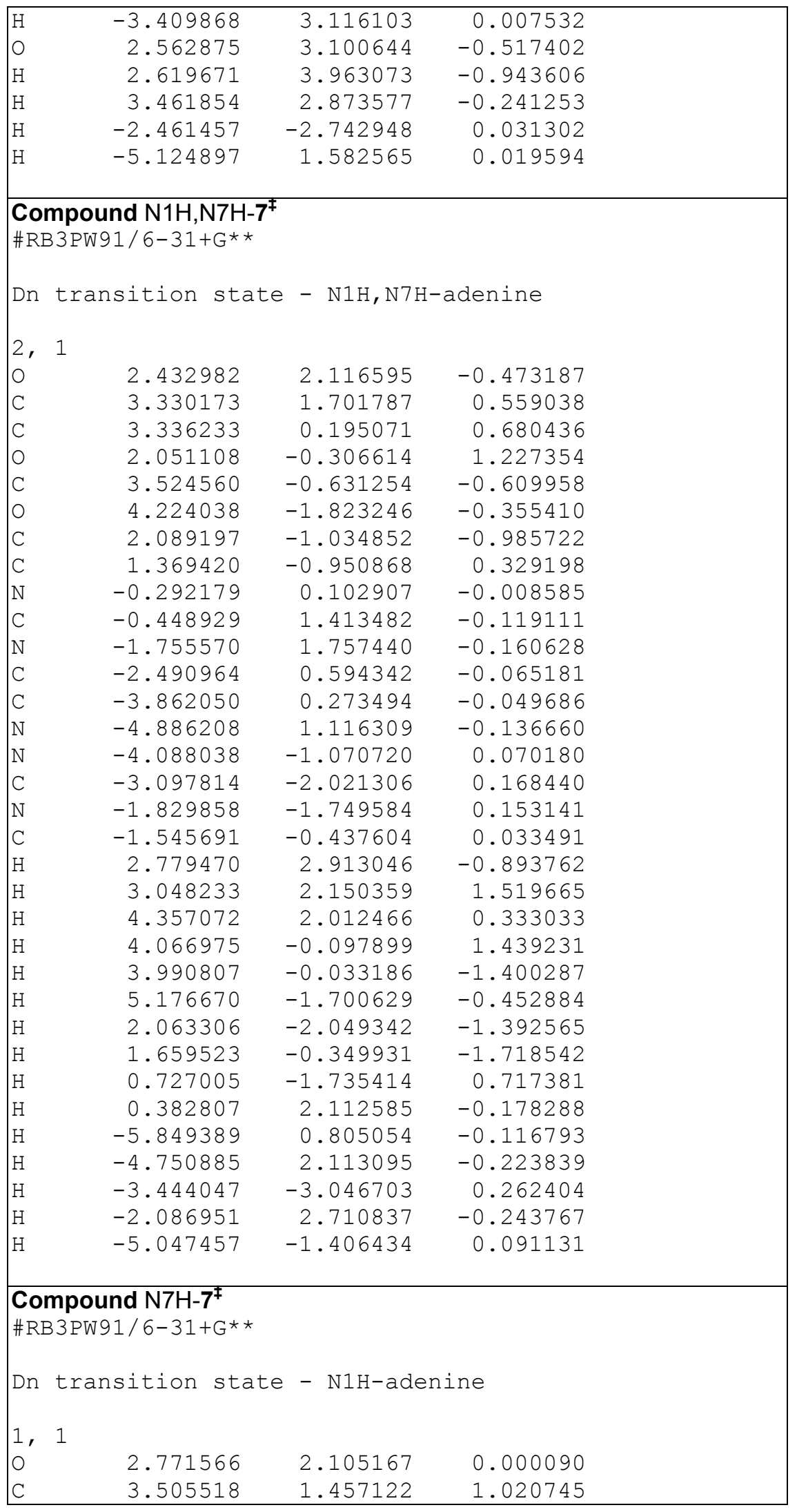


McCann \& Berti

page $\$ 20$

\begin{tabular}{|c|c|c|c|}
\hline C & 3.452994 & -0.033670 & 0.782182 \\
\hline 0 & 2.095824 & -0.547149 & 1.133703 \\
\hline $\mathrm{C}$ & 3.655603 & -0.515058 & -0.669892 \\
\hline 0 & 4.338778 & -1.746957 & -0.714539 \\
\hline C & 2.221355 & -0.777650 & -1.151807 \\
\hline C & 1.461756 & -0.926986 & 0.102597 \\
\hline $\mathrm{N}$ & -0.548127 & 0.667531 & -0.256891 \\
\hline C & -1.064968 & 1.856564 & -0.484046 \\
\hline $\mathrm{N}$ & -2.425038 & 1.865651 & -0.430622 \\
\hline C & -2.823330 & 0.570946 & -0.144475 \\
\hline C & -4.035897 & -0.123664 & 0.050780 \\
\hline $\mathrm{N}$ & -5.267054 & 0.426564 & -0.029973 \\
\hline $\mathrm{N}$ & -3.957274 & -1.434186 & 0.317497 \\
\hline C & -2.760583 & -2.026109 & 0.391494 \\
\hline $\mathrm{N}$ & -1.562121 & -1.467137 & 0.229131 \\
\hline C & -1.632921 & -0.154999 & -0.041067 \\
\hline $\mathrm{H}$ & 2.883733 & 3.058989 & 0.083361 \\
\hline $\mathrm{H}$ & 3.108216 & 1.681053 & 2.019909 \\
\hline $\mathrm{H}$ & 4.567255 & 1.744238 & 1.004375 \\
\hline $\mathrm{H}$ & 4.114757 & -0.554365 & 1.478170 \\
\hline $\mathrm{H}$ & 4.146075 & 0.256998 & -1.270109 \\
\hline H & 5.290726 & -1.607707 & -0.783520 \\
\hline $\mathrm{H}$ & 2.150516 & -1.670306 & -1.781767 \\
\hline $\mathrm{H}$ & 1.793581 & 0.067128 & -1.702560 \\
\hline $\mathrm{H}$ & 0.493660 & -1.418556 & 0.257862 \\
\hline $\mathrm{H}$ & -0.497042 & 2.752359 & -0.695309 \\
\hline $\mathrm{H}$ & -6.055738 & -0.167226 & 0.180508 \\
\hline $\mathrm{H}$ & -5.422663 & 1.416655 & -0.106365 \\
\hline $\mathrm{H}$ & -2.776911 & -3.090127 & 0.612707 \\
\hline H & -3.005388 & 2.674433 & -0.594346 \\
\hline \multicolumn{4}{|c|}{$\begin{array}{l}\text { Compound } \mathbf{\alpha}^{-8 \mathbf{a}^{\ddagger}} \\
\# \text { RB3PW91/6-31+G**}\end{array}$} \\
\hline \multicolumn{4}{|c|}{ An_TS - alpha-8a } \\
\hline \multicolumn{4}{|c|}{1,1} \\
\hline O & -0.044421 & -0.800260 & -0.915427 \\
\hline C & -0.784519 & -0.832331 & 0.104805 \\
\hline C & 1.003450 & 0.267117 & -0.772412 \\
\hline C & -0.511641 & 0.205040 & 1.101089 \\
\hline C & 0.446890 & 1.153986 & 0.361943 \\
\hline C & 2.305322 & -0.448162 & -0.486059 \\
\hline O & -0.324607 & 2.213051 & -0.146824 \\
\hline 0 & 2.165863 & -1.065953 & 0.776607 \\
\hline $\mathrm{H}$ & -1.555053 & -1.600495 & 0.130809 \\
\hline $\mathrm{H}$ & 1.019551 & 0.775066 & -1.738319 \\
\hline $\mathrm{H}$ & -0.050198 & -0.276006 & 1.975619 \\
\hline $\mathrm{H}$ & -1.436092 & 0.694883 & 1.426942 \\
\hline $\mathrm{H}$ & 1.255372 & 1.494802 & 1.015757 \\
\hline $\mathrm{H}$ & 3.103581 & 0.308629 & -0.494183 \\
\hline $\mathrm{H}$ & 2.512118 & -1.174129 & -1.282977 \\
\hline $\mathrm{H}$ & 2.934684 & -1.615632 & 0.969342 \\
\hline 0 & -3.596945 & -0.656988 & -0.112636 \\
\hline $\mathrm{H}$ & -4.008648 & -0.274726 & -0.896947 \\
\hline
\end{tabular}


McCann \& Berti

page S21

\begin{tabular}{|c|c|c|c|}
\hline $\begin{array}{l}\mathrm{H} \\
\mathrm{H}\end{array}$ & $\begin{array}{r}-4.331506 \\
0.200063\end{array}$ & $\begin{array}{r}-0.944538 \\
3.019443\end{array}$ & $\begin{array}{r}0.442834 \\
-0.218841\end{array}$ \\
\hline \multicolumn{4}{|c|}{$\begin{array}{l}\text { Compound } \boldsymbol{\alpha}_{-8 \mathbf{b}^{\ddagger}} \\
\# \text { RB3PW } 1 / 6-31+\mathrm{G}^{*}\end{array}$} \\
\hline An TS & - alpha-8b & & \\
\hline \multicolumn{4}{|l|}{1,1} \\
\hline 0 & 2.447112 & -0.365629 & 0.616508 \\
\hline C & 2.169442 & 0.101126 & -0.687684 \\
\hline C & 0.671971 & 0.288184 & -0.790667 \\
\hline 0 & 0.047404 & -1.070472 & -0.712744 \\
\hline C & -0.004480 & 1.054742 & 0.365260 \\
\hline 0 & -1.168701 & 1.729580 & -0.072025 \\
\hline C & -0.448052 & -0.075467 & 1.303413 \\
\hline C & -0.582226 & -1.210757 & 0.374694 \\
\hline $\mathrm{H}$ & 3.382859 & -0.583087 & 0.702660 \\
\hline $\mathrm{H}$ & 2.501788 & -0.604524 & -1.460112 \\
\hline $\mathrm{H}$ & 2.638030 & 1.075884 & -0.888794 \\
\hline $\mathrm{H}$ & 0.384181 & 0.670121 & -1.772251 \\
\hline $\mathrm{H}$ & 0.701579 & 1.731582 & 0.854063 \\
\hline $\mathrm{H}$ & -0.974901 & 2.651097 & -0.281829 \\
\hline $\mathrm{H}$ & -1.354795 & 0.140523 & 1.870060 \\
\hline $\mathrm{H}$ & 0.358125 & -0.344401 & 2.002571 \\
\hline $\mathrm{H}$ & -1.010293 & -2.189554 & 0.570958 \\
\hline O & -2.710664 & -0.638702 & -0.343531 \\
\hline $\mathrm{H}$ & -3.574417 & -1.052202 & -0.457344 \\
\hline $\mathrm{H}$ & -2.813289 & 0.319377 & -0.435740 \\
\hline \multicolumn{4}{|c|}{$\begin{array}{l}\text { Compound } \boldsymbol{\beta}_{-8^{\ddagger}}^{\ddagger} \\
\# \quad \text { RB3PW } 91 / 6-31+G^{* *}\end{array}$} \\
\hline An $T S$ & - alpha-8b & & \\
\hline \multicolumn{4}{|l|}{1,1} \\
\hline 0 & -0.564842 & 1.791053 & -0.731277 \\
\hline C & 0.510576 & 1.972155 & 0.167585 \\
\hline C & 1.019274 & 0.603883 & 0.556086 \\
\hline 0 & -0.054126 & -0.065972 & 1.369997 \\
\hline C & 1.270262 & -0.401882 & -0.588619 \\
\hline O & 2.318863 & -1.283906 & -0.273008 \\
\hline C & -0.022040 & -1.231761 & -0.611682 \\
\hline C & -0.580395 & -1.012205 & 0.723247 \\
\hline $\mathrm{H}$ & -0.862743 & 2.646393 & -1.063084 \\
\hline $\mathrm{H}$ & 0.208473 & 2.523344 & 1.068059 \\
\hline $\mathrm{H}$ & 1.353010 & 2.507781 & -0.293966 \\
\hline $\mathrm{H}$ & 1.867101 & 0.674119 & 1.240729 \\
\hline $\mathrm{H}$ & 1.426943 & 0.122209 & -1.536239 \\
\hline $\mathrm{H}$ & 3.157759 & -0.969384 & -0.631178 \\
\hline $\mathrm{H}$ & 0.150967 & -2.290934 & -0.830775 \\
\hline $\mathrm{H}$ & -0.773917 & -0.856957 & -1.322260 \\
\hline $\mathrm{H}$ & -1.376270 & -1.559568 & 1.220723 \\
\hline 0 & -3.083060 & -0.625623 & -0.211642 \\
\hline $\mathrm{H}$ & -3.867365 & -1.183517 & -0.282132 \\
\hline
\end{tabular}


McCann \& Berti

page $S 22$

\begin{tabular}{lllll}
\hline $\mathrm{H}$ & -3.404704 & 0.280960 & -0.282140
\end{tabular}

\section{References}

(1) Pagano, A. R.; Lajewski, W. M.; Jones, R. A. J. Am. Chem. Soc. 1995, 117, 11669-11672.

(2) Orji, C. C.; Kelly, J.; Ashburn, D. A.; Silks, L. A., III J. Chem. Soc., Perkin Trans. 1 1996, 595-597.

(3) Hove-Jensen, B.; Harlow, K. W.; King, C. J.; Switzer, R. L. J. Biol. Chem. 1986, 261, 6765-6771.

(4) Tolbert, T. J.; Williamson, J. R. J. Am. Chem. Soc. 1996, 118, 7929-7940.

(5) Gross, A.; Abril, O.; Lewis, J. M.; Geresh, S.; Whitesides, G. M. J. Am. Chem. Soc. 1983, $105,7428-7435$.

(6) Shi, W. X.; Tanaka, K. S. E.; Crother, T. R.; Taylor, M. W.; Almo, S. C.; Schramm, V. L. Biochemistry 2001, 40, 10800-10809.

(7) Booker, S.; Stubbe, J. Proc. Natl. Acad. Sci. U. S. A. 1993, 90, 8352-8356.

(8) Raap, J.; Vanboom, J. H.; Vanlieshout, H. C.; Haasnoot, C. A. G. J. Am. Chem. Soc. 1988, 110, 2736-2743.

(9) Gerlt, J. A.; Youngblood, A. V. J. Am. Chem. Soc. 1980, 102, 7433-7438.

(10) Saunders, M.; Laidig, K. E.; Wolfsberg, M. J. Am. Chem. Soc. 1989, 111, 8989-8994. 